\title{
On Tate's Proof of a Theorem of Dedekind
}

\author{
Shiv Gupta \\ Department of Mathematics, West Chester University, West Chester, PA, USA \\ Email: sguptai@wcupa.edu
}

How to cite this paper: Gupta, S. (2018) On Tate's Proof of a Theorem of Dedekind. Open Journal of Discrete Mathematics, 8, 73-78.

https://doi.org/10.4236/ojdm.2018.83007

Received: March 13, 2018

Accepted: June 29, 2018

Published: July 2, 2018

Copyright (c) 2018 by author and Scientific Research Publishing Inc. This work is licensed under the Creative Commons Attribution International License (CC BY 4.0).

http://creativecommons.org/licenses/by/4.0/

\author{
Abstract \\ In this note we give a complete proof of a theorem of Dedekind. \\ Keywords \\ Galois Group of a Polynomial
}

\section{Introduction}

In this note we give a complete proof of the following theorem of Dedekind. Our proof is a somewhat detailed version of the one given in Basic Algebra by Jacobson, Volume I, [1] and we shall keep the notations used in that proof.

Theorem 1 Let $f(x) \in \mathbb{Z}[x]$ be square-free monic polynomial of degree $n$ and $p$ be a prime such that $p$ does not divide the discriminant of $f(x)$. Let $G \subset S_{n}$ be the Galois group of $f(x)$ over the field $\mathbb{Q}$ of rational numbers. Suppose that $f_{p}=\bar{f}=f(\bmod p) \in \mathbb{Z}_{p}[x]$ factors as:

$$
f_{p}=\bar{f}=\prod_{i=1}^{r} \overline{f_{i}}
$$

where $\bar{f}_{i}$ are distinct monic irreducible polynomials in $\mathbb{Z}_{p}[x]$, degree $\left(\overline{f_{i}}\right)=d_{i}, \quad 1 \leq i \leq r$, and $d_{1}+d_{2}+\cdots+d_{r}=n$.

Then there exists an automorphism $\sigma \in G$ which when considered as a permutation on the zeros of $f(x)$ is a product of disjoint cycles of lengths $d_{1}, d_{2}, \cdots, d_{r}$.

\section{Preliminary Results}

We shall assume that the reader is familiar with the following well-known results.

1) Let $\mathbb{F}$ be a field and $f(x) \in \mathbb{F}[x]$ be a polynomial of degree $n \geq 2$. Then any two splitting fields of $f(x)$ are isomorphic.

2) A finitely generated Abelian group is direct sum of (finitely many) cyclic 
groups. (This is the fundamental theorem of finitely generated Abelian groups).

3) A system of $n$ homogeneous equation in $m>n$ variables has a non-trivial solutions.

4) Let $\mathbb{E} / \mathbb{F}$ be an algebraic extension. Then any subring of $\mathbb{E}$ containing $\mathbb{F}$ is a subfield of $\mathbb{E}$. Proof: Let $K$ be a ring such that $\mathbb{F} \subset K \subset \mathbb{E}$. Let $\alpha \in K-\mathbb{F}$. As $\alpha$ is algebraic over $\mathbb{F}, \mathbb{F}(\alpha)=\mathbb{F}[\alpha]$. So $\alpha^{-1} \in \mathbb{F}(\alpha) \subset K$.

5) (Dedekind's Independence Theorem). Distinct characters of a monoid (a set with associative binary operation with an identity element) into a field are linearly independent. That is if $\chi_{1}, \chi_{2}, \cdots, \chi_{n}$ are distinct characters of a monoid into a field $\mathbb{F}$, then the only elements $a_{i} \in \mathbb{F}, 1 \leq i \leq n$, such that

$$
a_{1} \chi_{1}(h)+a_{2} \chi_{2}(h)+\cdots+a_{n} \chi_{n}(h)=0
$$

for all $h \in H$ are $a_{i}=0,1 \leq i \leq n$.

6) Let $p$ be a prime and $G F\left(p^{m}\right)$ be a finite field with $p^{m}$ elements. Then the group $\operatorname{Aut}\left(G F\left(p^{m}\right)\right)=\langle\sigma\rangle$ is cyclic of order $m$ and the generating automorphism $\sigma$ maps $\alpha \in G F\left(p^{m}\right)$ to $\alpha^{p}$.

7) If $R$ is a commutative ring with identity and $M$ is a maximal ideal of $R$ then $R / M$ is a field.

8) Let $\sigma, \eta \in S_{n}$. Then $\sigma$ and $\eta^{-1} \sigma \eta$ have same cyclic structure.

Let $f(x) \in \mathbb{Z}[x]$ be a polynomial of degree $n \geq 1$, and $p$ a prime number. Then $f_{p}(x) \in \mathbb{Z}_{p}[x]$ will denote the polynomial obtained by reducing the coefficients of $f(x)$ modulo $p$.

Theorem 2 Let $f(x) \in \mathbb{Z}[x]$ be a monic polynomial of degree $n \geq 1$ and $p$ be a prime number which does not divide the discriminant of $f(x)$. Let $\mathbb{E}$ be a splitting field of $f(x)$ over $\mathbb{Q}$. Let $\mathbb{E}_{p}$ be a splitting field of $f_{p}(x)$ over $\mathbb{Z}_{p}=\mathbb{Z} /(p)$. Let

$$
\begin{gathered}
f(x)=\left(x-r_{1}\right)\left(x-r_{2}\right) \cdots\left(x-r_{n}\right), \quad r_{i} \in \mathbb{E} \subset \mathbb{C}, 1 \leq i \leq n \\
R=\left\{r_{1}, r_{2}, \cdots, r_{n}\right\}, \\
R_{p}=\left\{\overline{r_{1}}, \overline{r_{2}}, \cdots, \overline{r_{n}}\right\} \subset \mathbb{E}_{p}
\end{gathered}
$$

where $\bar{r}_{i}, \quad 1 \leq i \leq n$ are the roots of $f_{p}(x) \in \mathbb{Z}_{p}[x]$ and

$$
\mathbb{E}=\mathbb{Q}\left(r_{1}, r_{2}, \cdots, r_{n}\right), \mathbb{E}_{p}=\mathbb{Z}_{p}\left(\overline{r_{1}}, \overline{r_{2}}, \cdots, \overline{r_{n}}\right)
$$

Let $D=\mathbb{Z}\left[r_{1}, r_{2}, \cdots, r_{n}\right]$ be the subring generated by the roots of $r_{1}, r_{2}, \cdots, r_{n}$ of $f(x)$ in $\mathbb{C}$. Then

-1) There exists a homomorphism $\psi$ of Donto $\mathbb{E}_{p}$.

-2) Any such homomorphism $\psi$ gives a bijection of the set $R$ of the roots of $f(x)$ in $\mathbb{E}$ onto the set $R_{p}$ of the roots of the $f_{p}(x)$ in $\mathbb{E}_{p}$.

- 3) If $\psi$ and $\psi^{\prime}$ are two such homomorphisms then there exist $\sigma \in \operatorname{Aut}(\mathbb{E} / \mathbb{Q})=\operatorname{Gal}(f(x))$, such that $\psi^{\prime}=\psi \cdot \sigma$. (Note that the restriction of $\sigma$ to $D$ is an automorphism of $D)$.

Proof 1) One has that:

$$
\mathbb{E}=\mathbb{Q}\left(r_{1}, r_{2}, \cdots, r_{n}\right)=\mathbb{Q}\left[r_{1}, r_{2}, \cdots, r_{n}\right]
$$


We claim that $D=\mathbb{Z}\left[r_{1}, r_{2}, \cdots, r_{n}\right]$ is a finitely generated (additive) Abelian group. Since each $r_{i}$ is a root of the monic polynomial $f(x) \in \mathbb{Z}[x]$ of degree $n$ any positive power of $r_{i}, 1 \leq i \leq n$ can be expressed as an integral linear combination of $1, r_{i}, r_{i}^{2}, \cdots r_{i}^{n-1}$. It follows that

$$
D=\sum_{0 \leq e_{i} \leq n-1} \mathbb{Z} r_{1}^{e_{1}} r_{2}^{e_{2}} \cdots r_{n}^{e_{n}}
$$

Therefore $D$ is a finitely generated (additive) Abelian group generated by at most $n^{n}$ elements. By the Fundamental Theorem for Finitely Generated Abelian Groups $D$ is a direct sum of finitely many cyclic groups. Since $D \subset \mathbb{C}$, none of these cyclic groups is finite. So $D$ is a direct sum of finitely many infinite cyclic groups. Let $\left\{u_{1}, u_{2}, \cdots, u_{N}\right\}$ be a set consisting of an independent generating system of $D$. We have

$$
D=\mathbb{Z} u_{1} \oplus \mathbb{Z} u_{2} \oplus \cdots \oplus \mathbb{Z} u_{N}, \quad N \leq n^{n} .
$$

We claim that $\left\{u_{1}, u_{2}, \cdots, u_{N}\right\}$ is a basis of $\mathbb{E} / \mathbb{Q}$. Obviously $\left\{u_{1}, u_{2}, \cdots, u_{N}\right\}$ is linearly independent over $\mathbb{Q}$. Let $\mathbb{Q} D=\sum_{1 \leq i \leq N} \mathbb{Q} u_{i}$. Then $\mathbb{Q} D$ is a ring and $\mathbb{Q} \subset \mathbb{Q} D \subset \mathbb{E}$ therefore $\mathbb{Q} D$ is a field. Since $r_{i} \in D$ for $1 \leq i \leq n$, by (4) $\mathbb{Q} D=\mathbb{E}$ and $\left\{u_{1}, u_{2}, \cdots, u_{N}\right\}$ is a basis of $E / \mathbb{Q}$. As $D=\mathbb{Z} u_{1} \oplus \mathbb{Z} u_{2} \oplus \cdots \oplus \mathbb{Z} u_{N}$,

$$
p D=\mathbb{Z}\left(p u_{1}\right) \oplus \mathbb{Z}\left(p u_{2}\right) \oplus \cdots \oplus \mathbb{Z}\left(p u_{N}\right)
$$

is an ideal of $D$ and

$$
D / p D=\left\{\overline{a_{1} u_{1}+a_{2} u_{2}+\cdots+a_{N} u_{N}}: 0 \leq a_{i} \leq p-1\right\} .
$$

Therefore the $D / p D$ is finite of order $p^{N}$. Let $M$ be a maximal ideal of $D$ containing $p D$. That is $p D \subset M \subset D$ and $D / M$ is a finite field of characteristic $p$ and so it has a subfield isomorphic to $\mathbb{Z}_{p}=\mathbb{Z} / p \mathbb{Z}$ which we will identify as $\mathbb{Z}_{p}$ in what follows. As

$$
D / M \approx \frac{D / p D}{M / p D}
$$

the order of $D / M$ is $p^{m}, 1 \leq m \leq N$. Consider the canonical epimorphism

$$
v: D \rightarrow D / M
$$

whose kernel is $M$ and $p \mathbb{Z} \subset M$. Therefore $v(\mathbb{Z})=\mathbb{Z}_{p}$. We note that as $D=\mathbb{Z}\left[r_{1}, r_{2}, \cdots, r_{n}\right]$ we have for $1 \leq i \leq n$

$$
v\left(r_{i}\right)=r_{i}+M=\overline{r_{i}}, \quad v(D)=\mathbb{Z}_{p}\left[\overline{r_{1}}, \overline{r_{2}}, \cdots, \overline{r_{n}}\right]
$$

As $v$ is an epimorphism we have

$$
v(D)=D / M=\mathbb{Z}_{p}\left[\overline{r_{1}}, \overline{r_{2}}, \cdots, \overline{r_{n}}\right]
$$

is a splitting field of $f_{p}(x)$ over $\mathbb{Z}_{p}$. As both $D / M$ and $\mathbb{E}_{p}$ are splitting fields of $f_{p}(x)$ over $\mathbb{Z}_{p}$ they are isomorphic. Let

$$
\phi: D / M \rightarrow \mathbb{Z}_{p}
$$

be such an isomorphism. Then $\psi=\phi \cdot v$ is a homomorphism of $D$ onto $\mathbb{E}_{p}$. 
2) Let $\psi: D \rightarrow \mathbb{E}_{p}$ be a homomorphism. So $\psi(1)=1$. As $\mathbb{Z} \subset D$, and $\mathbb{E}_{p}$ has characteristic $p, \psi(p)=0$, so $\psi(\mathbb{Z})=\mathbb{Z}_{p} \subset \mathbb{E}_{p} \cdot \psi$ can be extended to a homomorphism of the polynomial rings $D[x] \rightarrow \mathbb{E}_{p}[x]$. Under this mapping $f(x) \rightarrow f_{p}(x)$. As

$$
\begin{gathered}
f(x)=\left(x-r_{1}\right)\left(x-r_{2}\right) \cdots\left(x-r_{n}\right) \\
\psi(f(x))=f_{p}(x)=\left(x-\psi\left(r_{1}\right)\right)\left(x-\psi\left(r_{2}\right)\right) \cdots\left(x-\psi\left(r_{n}\right)\right),
\end{gathered}
$$

$\psi\left(r_{i}\right), 1 \leq i \leq n$ are the roots of the $f_{p}(x)$ in $\mathbb{E}_{p}$ and therefore the restriction of $\psi$ to $R$

$$
\psi_{\mid R}:\left\{r_{1}, r_{2}, \cdots, r_{n}\right\} \rightarrow\left\{\overline{r_{1}}, \overline{r_{2}}, \cdots, \overline{r_{n}}\right\}
$$

is a bijection of the set $R$ of roots of $f(x)$ in $\mathbb{E}$ to the set $R_{p}$ of the roots of $f_{p}(x)$ in $\mathbb{E}_{p}$.

3) We have seen that given a homomorphism $\psi: D \rightarrow \mathbb{E}_{p}$, and $\sigma \in \operatorname{Gal}(f)=\operatorname{Aut}(\mathbb{E} / \mathbb{Q}), \quad \psi^{\prime}=\psi \cdot \sigma$ is also a homomorphism from $D$ to $\mathbb{E}_{p}$. We note that the restriction of $\sigma \in \operatorname{Aut}(\mathbb{E} / \mathbb{Q})$ to $D=\mathbb{Z}\left[r_{1}, r_{2}, \cdots, r_{n}\right]$ is also an automorphism of the ring $D$. Since $[\mathbb{E}: \mathbb{Q}]=N$, the group $\operatorname{Aut}(\mathbb{E} / \mathbb{Q})$ has order $N$. Let

$$
\operatorname{Aut}(\mathbb{E} / \mathbb{Q})=\left\{\sigma_{1}, \sigma_{2}, \cdots, \sigma_{N}\right\}
$$

So given a non-trivial homomorphism $\psi: D \rightarrow \mathbb{E}_{p}$, we get $N$ distinct homomorphisms $\psi_{j}=\psi \cdot \sigma_{j}, 1 \leq j \leq N$, from $D$ to $\mathbb{E}_{p}$. We claim that these are all the homomorphisms from $D$ to $\mathbb{E}_{p}$. Suppose that there is a homomorphism from $D$ to $\mathbb{E}_{p}$ which is different from $\psi_{j}, 1 \leq j \leq N$. Let us denote it by $\psi_{N+1}$. By Dedekind Independence Theorem the set $\left\{\psi_{1}, \psi_{2}, \cdots, \psi_{N}, \psi_{N+1}\right\}$ of $N+1$ homomorphisms from $D$ to $\mathbb{E}_{p}$ is linearly independent over the field $\mathbb{E}_{p}$.

Consider the following system of $N$ homogeneous equations in $N+1$ variables $\left\{x_{1}, x_{2}, \cdots, x_{N}, x_{N+1}\right\}$,

$$
\sum_{i=1}^{N+1} x_{i} \psi_{i}\left(u_{j}\right)=0, \quad 1 \leq j \leq N .
$$

Since there are more variables than the equations this system of equations has a non-trivial solution. Let this non-trivial solution be $x_{i}=a_{i} \in \mathbb{E}_{p}, \quad 1 \leq i \leq N+1$. So we have

$$
\sum_{i=1}^{i=N+1} a_{i} \psi_{i}\left(u_{j}\right)=0, \quad 1 \leq j \leq N .
$$

Let $y \in D=\mathbb{Z} u_{1} \oplus \mathbb{Z} u_{2} \oplus \cdots \oplus \mathbb{Z} u_{N}$. So $y=n_{1} u_{1}+n_{2} u_{2}+\cdots+n_{N} u_{N}, \quad n_{k} \in \mathbb{Z}$, $1 \leq k \leq N$. Then for $1 \leq i \leq N+1$ we have

$$
\psi_{i}(y)=\overline{n_{1}} \psi_{i}\left(u_{1}\right)+\overline{n_{2}} \psi_{i}\left(u_{2}\right)+\cdots+\overline{n_{N}} \psi_{i}\left(u_{N}\right)=\sum_{j=1}^{j=N} \overline{n_{j}} \psi_{i}\left(u_{j}\right)
$$

where $\overline{n_{j}}=n_{j}+(p)$. We shall show that

$$
\sum_{i=1}^{i=N+1} a_{i} \psi_{i}(y)=0
$$


which will contradict the linear independence of $\left\{\psi_{1}, \psi_{2}, \cdots, \psi_{N}, \psi_{N+1}\right\}$ over $\mathbb{E}_{p}$.

$$
\begin{aligned}
& \sum_{i=1}^{i=N+1} a_{i} \psi_{i}(y) \\
= & \sum_{i=1}^{i=N+1} a_{i} \sum_{j=1}^{j=N} \overline{n_{j}} \psi_{i}\left(u_{j}\right) \\
= & \sum_{i=1}^{i=N+1} a_{i}\left(\overline{n_{1}} \psi_{i}\left(u_{1}\right)+\overline{n_{2}} \psi_{i}\left(u_{2}\right)+\cdots+\overline{n_{N}} \psi_{i}\left(u_{N}\right)\right) \\
= & \bar{n}_{1} \sum_{i=1}^{i=N+1} a_{i} \psi_{i}\left(u_{1}\right)+\overline{n_{2}} \sum_{i=1}^{i=N+1} a_{i} \psi_{i}\left(u_{2}\right)+\cdots+\overline{n_{N}} \sum_{i=1}^{i=N+1} a_{i} \psi_{i}\left(u_{N}\right) \\
= & 0 .
\end{aligned}
$$

\section{Proof of the Main Theorem}

Since the field $\mathbb{E}_{p}$ has order $p^{m}$, the group $\operatorname{Aut}\left(\mathbb{E}_{p}\right)$ has order $m$ and $\pi: \mathbb{E}_{p} \rightarrow \mathbb{E}_{p}$ where $\pi(a)=a^{p}$ for all $a \in \mathbb{E}_{p}$, is the generating automorphism of $\operatorname{Aut}\left(\mathbb{E}_{p}\right)$. So if $\psi: D \rightarrow \mathbb{E}_{p}$ is any homomorphism then so is $\pi \cdot \psi$. Since $\psi$ and $\pi \cdot \psi$ are two homomorphisms from $D$ to $\mathbb{E}_{p}$ there exist $\sigma \in \operatorname{Aut}(\mathbb{E} / \mathbb{Q})$ such that $\pi \cdot \psi=\psi \cdot \sigma$ or $\psi^{-1} \cdot \pi \cdot \psi=\sigma$. This proves that the action on $\sigma$ on $\left\{r_{1}, r_{2}, \cdots, r_{n}\right\}$ is similar to the action of $\pi$ on $\left\{\bar{r}_{1}, \bar{r}_{2}, \cdots, \overline{r_{n}}\right\}$. Note: In the following diagram the mapping

$$
D \stackrel{\sigma}{\longrightarrow} D
$$

is the restriction of $\sigma \in \operatorname{Aut}(\mathbb{E} / \mathbb{Q})$ to $D$ and we are only concerned with the effect of the mappings $\sigma, \psi$ and $\pi$ on $\left\{r_{1}, r_{2}, \cdots, r_{n}\right\}$ and $\left\{\bar{r}_{1}, \overline{r_{2}}, \cdots, \overline{r_{n}}\right\}$. Clearly

$$
\begin{gathered}
\left\{r_{1}, r_{2}, \cdots, r_{n}\right\} \stackrel{\sigma}{\longrightarrow}\left\{r_{1}, r_{2}, \cdots, r_{n}\right\} \\
\left\{\overline{r_{1}}, \overline{r_{2}}, \cdots, \overline{r_{n}}\right\} \stackrel{\pi}{\longrightarrow}\left\{\overline{r_{1}}, \bar{r}_{2}, \cdots, \overline{r_{n}}\right\} \\
\left\{r_{1}, r_{2}, \cdots, r_{n}\right\} \stackrel{\psi}{\longrightarrow}\left\{\overline{r_{1}}, \overline{r_{2}}, \cdots, \overline{r_{n}}\right\} \\
D \stackrel{\sigma}{\longrightarrow} D \\
\psi \\
\downarrow \downarrow \\
\mathbb{E}_{p} \stackrel{\pi}{\longrightarrow} \mathbb{E}_{p}
\end{gathered}
$$

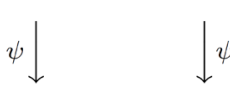

As $\psi^{-1} \cdot \pi \cdot \psi=\sigma$ and $\psi \cdot \sigma \cdot \psi^{-1}=\pi$ the effect of $\sigma$ on $\left\{r_{1}, r_{2}, \cdots, r_{n}\right\}$ is similar to the effect of $\pi$ on $\left\{\bar{r}_{1}, \overline{r_{2}}, \cdots, \overline{r_{n}}\right\}$. This is further illustrated by the following:

$$
\begin{gathered}
\sigma\left(r_{i}\right)=r_{j} \Rightarrow \pi\left(\overline{r_{i}}\right)=\bar{r}_{j} \\
\bar{r}_{i} \stackrel{\psi^{-1}}{\longrightarrow} r_{i} \stackrel{\sigma}{\longrightarrow} r_{j} \stackrel{\psi}{\longrightarrow} \overline{r_{j}} \\
\pi\left(\overline{r_{i}}\right)=\bar{r}_{j} \Rightarrow \sigma\left(r_{i}\right)=r_{j} \\
r_{i} \stackrel{\psi}{\longrightarrow} \bar{r}_{i} \stackrel{\pi}{\longrightarrow} \bar{r}_{j} \stackrel{\psi^{-1}}{\longrightarrow} r_{j}
\end{gathered}
$$




\section{References}

[1] Jacobson, N. (2014) Basic Algebra. 2nd Edition, Dover Publications, Inc., Mineola, New York. 